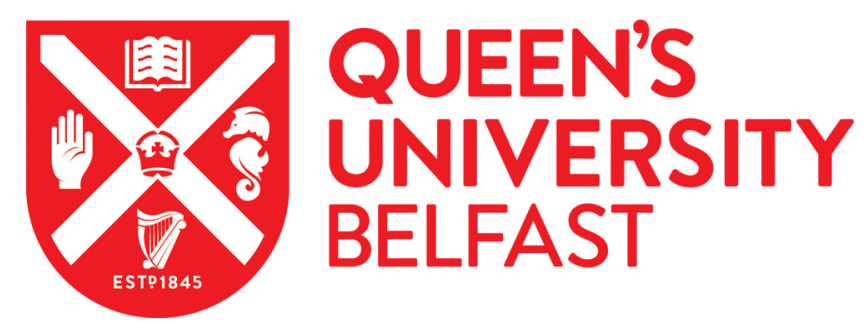

\title{
Channel Correlation Diversity in MU-MIMO Systems - Analysis and Measurements
}

Tataria, H., Sangodoyin, S., Molisch, A. F., Smith, P. J., Matthaiou, M., Zhang , J., \& Thoma, R. S. (2019). Channel Correlation Diversity in MU-MIMO Systems - Analysis and Measurements. In 2019 IEEE 30th Annual International Symposium on Personal, Indoor and Mobile Radio Communications (PIMRC) (IEEE Annual International Symposium on Personal, Indoor and Mobile Radio Communications (PIMRC): Proceedings). Institute of Electrical and Electronics Engineers Inc.. https://doi.org/10.1109/PIMRC.2019.8904875

Published in:

2019 IEEE 30th Annual International Symposium on Personal, Indoor and Mobile Radio Communications (PIMRC)

Document Version:

Peer reviewed version

Queen's University Belfast - Research Portal:

Link to publication record in Queen's University Belfast Research Portal

Publisher rights

(c) 2018 IEEE. This work is made available online in accordance with the publisher's policies. Please refer to any applicable terms of use of the publisher.

\section{General rights}

Copyright for the publications made accessible via the Queen's University Belfast Research Portal is retained by the author(s) and / or other copyright owners and it is a condition of accessing these publications that users recognise and abide by the legal requirements associated with these rights.

Take down policy

The Research Portal is Queen's institutional repository that provides access to Queen's research output. Every effort has been made to ensure that content in the Research Portal does not infringe any person's rights, or applicable UK laws. If you discover content in the Research Portal that you believe breaches copyright or violates any law, please contact openaccess@qub.ac.uk. 


\title{
Channel Correlation Diversity in Multiuser Systems
}

\author{
Harsh Tataria*, Seun Sangodoyin ${ }^{\dagger}$, Andreas F. Molisch ${ }^{\dagger}$, Peter J. Smith ${ }^{\ddagger}$, Michail Matthaiou*, \\ Jianzhong Zhang ${ }^{\S}$, and Reiner. S. Thomä \\ *Institute of Electronics, Communications and Information Technology (ECIT), Queen's University Belfast, Belfast, U.K. \\ ${ }^{\dagger}$ Ming Hsieh Department of Electrical Engineering, University of Southern California, Los Angeles, CA, U.S.A.

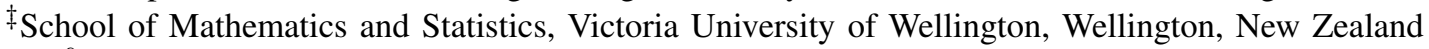 \\ $\S$ Mobility and Innovation Laboratory, Samsung Research America, Richardson, TX, U.S.A. \\ IInstitut für Informationstechnik, Technische Universität Ilmenau, Ilmenau, Germany \\ e-mail:\{h.tataria, m.matthaiou\}@qub.ac.uk, \{sangodoy, molisch\}@usc.edu, peter.smith@vuw.ac.nz, \\ jianzhong.z@samsung.com, and reiner.thomae@tu-ilmenau.de
}

\begin{abstract}
In multiuser multiple-input multiple-output (MUMIMO) systems, channel correlation is detrimental to the system performance. We demonstrate that the widely used, yet overly simplified, correlation models that generate identical correlation profiles for each terminal tend to severely underestimate the system performance. In sharp contrast, more physically motivated models that capture variations in the power angular spectra across multiple terminals, generate diverse correlation patterns. This has a significant impact on the system performance. Assuming correlated Rayleigh fading and downlink zero-forcing precoding, tight closed-form approximations corroborated to the average signal-to-noise-ratio, and ergodic sum spectral efficiency are derived. Our expressions provide clear insights into the impact of diverse correlation patterns on the above performance metrics. Unlike previous works, the correlation models are parameterized with measured data from a recent $2.53 \mathrm{GHz}$ urban macrocellular campaign in Cologne, Germany. Overall, results from this paper can be treated as a timely re-calibration of performance expectations from practical MU-MIMO systems.
\end{abstract}

\section{INTRODUCTION}

In multiuser multiple-input multiple-output (MU-MIMO) systems, an antenna array at a cellular base station (BS) serves multiple user terminals. Typically, propagation between the array and a given terminal is characterized by the farfield multipath components (MPCs) arriving at the terminal from a cluster of scatterers [1]. Depending on the severity of scattering and the relative physical separation of terminals, MPCs often arrive at multiple terminals via the same clusters of scatterers, and hence are correlated [2,3]. Indeed, it is known from MU-MIMO literature, that channel correlation is detrimental to the terminal and the system spectral efficiency (see e.g., [1-5]). However, this finding is routinely reported when each terminal in the system has an identical correlation profile. In contrast, a different line of investigations has identified that correlation can enhance MU-MIMO performance [6-11]. The critical observation from these studies is that the departing spread of energy from the BS can arrive at a given terminal via a partially different set of clusters located within the vicinity of the terminal. This leads to variations in the power angular spectrum, and hence the statistics of the channel. Fundamentally, such variations depend on the geometry of scattering, as well as the inter-element spacing at the BS. To capture the above physical manifestations, models such as one-ring correlation have been proposed [6-9]. These models are characterized in terms of the mean azimuth directionof-arrival (DOA) at a terminal, departing azimuth angular spread, as well as the antenna spacing at the BS. The works in $[7,8]$ utilized the one-ring model to group the terminals with similar correlation characteristics in order to motivate a novel multiuser processing technique. The authors in [9] report that if the terminal correlation matrices span orthogonal subspaces, the fundamental impairment of pilot contamination vanishes, allowing the ergodic sum spectral efficiency to grow without bound with the number of BS antennas. The ergodic spectral efficiency of MU-MIMO systems was numerically investigated in [11] as a function of the azimuth angular spread with the presence of pilot contamination.

Despite the above efforts, it remains to be seen just how much performance gain is available with correlation diversity, relative to the case when each terminal has an identical correlation pattern. More importantly, almost all of the analytical results estimating MU-MIMO performance with correlation diversity, are left in terms of complex mathematical expressions and numerical fixed point algorithms, making it difficult to gain any practical insights into their behavior (see e.g., $[6,8,12,13])$. To gain a fundamental understanding of MUMIMO performance with and without correlation diversity, it is desirable to have an insightful and simple downlink performance measure. This is missing from the vast MUMIMO literature, and hence is the aim of the paper. Keeping this in mind, we derive simple, closed-form approximations to the downlink zero-forcing (ZF) expected signal-to-noiseratio (SNR) and ergodic sum spectral efficiency. As the more physical correlation models rely on the propagation channel's spatial parameters, for the most accurate parameterization, we extract the required model parameters from a recent $2.53 \mathrm{GHz}$ MU-MIMO measurement campaign in Cologne, Germany. To the best of the our knowledge, studies which use measured multipath parameters to investigate the diversity of correlation profiles in multiuser systems are rare. ${ }^{1}$

Specifically, the major contributions are as follows: Assuming spatially correlated Rayleigh fading, our ZF approximations provide clear insights into the impact of correlation diversity, as well as other system parameters, such as the number of BS antennas, number of terminals, and the average operational downlink SNR. We provide explicit insights into the fact that

\footnotetext{
${ }^{1}$ Note that exceptions are made for studies such as $[2,10]$, in which authors make initial investigations into characterizing the commonality of MPCs as a function of inter-terminal distance relative to the BS array.
} 
fixed correlation profiles tends to increase the expected ZF noise power, unlike diverse correlation profiles. As a result, we argue that fixed correlation provides a useful lower bound on the resulting MU-MIMO performance. Our numerical findings suggest that the choice of a particular correlation model has a significant impact on the expected ZF SNR and ergodic spectral efficiency. Physically motivated models, such as onering correlation, give enhanced performance over simplified models, such as the exponential and Clerckx correlation [5, 14]. To parameterize the one-ring model, we utilize measured angular spreads and mean DOA distributions at $2.53 \mathrm{GHz}$ from an urban macrocellular (UMa) campaign in Cologne.

Notation. Upper and lower boldface letters represent matrices and vectors. The $M \times M$ identity matrix is denoted as $\mathbf{I}_{M}$. Transpose, Hermitian transpose, inverse and trace operators are denoted by $(\cdot)^{T},(\cdot)^{H},(\cdot)^{-1}$, and $\operatorname{Tr}[\cdot]$, respectively. Moreover, $\|\cdot\|_{F}$ denotes the Frobenius norm. We use $\mathbf{h} \sim \mathcal{C N}(\mathbf{m}, \mathbf{R})$ to denote a complex Gaussian distribution for $\mathbf{h}$ with mean $\mathbf{m}$ and covariance matrix $\mathbf{R}$. Similarly, $h \sim \mathcal{U}[a, b]$ is used to denote a uniform random variable for $h$, taking on values from $a$ to $b$. Finally, $\mathbb{E}\{\cdot\}$ denotes the statistical expectation.

\section{SYSTEM MODEL}

We consider the downlink of a single-cell MU-MIMO system operating in an UMa environment. The BS is located at the center of a circular cell with radius $R_{\mathrm{c}}$, and is equipped with an array of $M$ transmit antennas. The BS serves $L$ singleantenna terminals $(M \geq L)$ in the same time-frequency resource. Channel knowledge is assumed at the BS with narrowband transmission and uniform power allocation.

Remark 1. At first sight, the assumption of perfect channel knowledge may seem rather naive. However, there are several reasons for this: Firstly, unlike previous studies, the central focus of the work is to devise a simple, yet accurate, performance metric to gain insights into the behavior of correlation diversity in multiuser systems. In contrast to prior studies, measured spatial parameters of the channel are utilized to capture the power angular spectra variations across multiple terminals. Under this heterogeneous scenario, it is extremely difficult to make analytical progress without perfect channel knowledge. Secondly, this assumption is more plausible in scenarios with low terminal mobility, where using time-division duplexing, a larger fraction of the channel coherence interval can be spent for uplink training. Thirdly, it is worth noting that the results obtained from the subsequent analysis and evaluations can be treated as a useful upper bound on the performance which may be seen in practice with imperfect channel knowledge.

\section{A. Propagation Channel and Received Signal}

The $1 \times M$ propagation channel to terminal $\ell$ from the BS array is denoted by $\mathbf{h}_{\ell}$, which is assumed to follow a spatially correlated Rayleigh fading distribution, i.e., $\mathbf{h}_{\ell} \sim \mathcal{C N}\left(\mathbf{0}, \mathbf{R}_{\ell}\right)$. Unlike previously (see e.g., [4,5]), $\mathbf{R}_{\ell}$, the $M \times M$ correlation matrix, is specific to terminal $\ell$. Naturally, $\mathbf{R}_{\ell}$ will be a function of the channel's spatial parameters [2,6-9]. For clarity, further discussion on the possible structures of $\mathbf{R}_{\ell}$ is deferred till Sec. V. The received signal at terminal $\ell$ is

$$
y_{\ell}=\sqrt{\frac{\rho_{\mathrm{t}} \beta_{\ell}}{\eta}} \mathbf{h}_{\ell} \mathbf{g}_{\ell} s_{\ell}+\sum_{\substack{i=1 \\ i \neq \ell}}^{L} \sqrt{\frac{\rho_{\mathrm{t}} \beta_{\ell}}{\eta}} \mathbf{h}_{\ell} \mathbf{g}_{i} s_{i}+n_{\ell},
$$

where $\rho_{\mathrm{t}}$ is the average transmit power at the BS and $\beta_{\ell}$ denotes the link gain of terminal $\ell$ (discussed later in the text). Furthermore, $\mathbf{g}_{\ell}$ is the $M \times 1$ un-normalized downlink precoding vector from the BS array to terminal $\ell$, obtained from $\ell$-th column of $\mathbf{G}$, the composite $M \times L$ un-normalized precoding matrix. The data symbol for terminal $\ell$ is denoted by $s_{\ell}$, such that $\mathbb{E}\left\{\left|s_{\ell}\right|^{2}\right\}=1, \forall \ell=1,2, \ldots, L$, and $n_{\ell} \sim \mathcal{C N}\left(0, \sigma^{2}\right)$ models the additive white Gaussian noise at terminal $\ell$. Note that $\sigma^{2}$ is fixed for all terminals $1,2, \ldots, L$. Following $[5,13]$, $\eta=\|\mathbf{G}\|_{F}^{2} / L$ is the precoding normalization parameter such that $\mathbb{E}\left\{\left\|\mathbf{g}_{\ell}\right\|^{2}\right\}=1$, for $\ell=1,2, \ldots, L$ (discussed further in the text). The link gain at terminal $\ell, \beta_{\ell}=A \zeta_{\ell}\left(r_{0} / r_{\ell}\right)^{\alpha}$ is composed of the large-scale propagation effects: Particularly, $A$ is the unit-less constant for geometric attenuation at a reference distance $r_{0}, r_{\ell}$ is the link distance between the BS and terminal $\ell, \alpha$ is the attenuation exponent and $\zeta_{\ell}$ models the effects of shadow fading which follows a lognormal distribution, i.e., $10 \log _{10}\left(\zeta_{\ell}\right) \sim \mathcal{N}\left(0, \sigma_{\text {sh }}^{2}\right)$. For clarity, we delay quoting values for the above parameters to Sec. V.

\section{B. ZF SNR and Ergodic Sum Spectral Efficiency}

It is well known that $\mathrm{ZF}$ precoding nulls multiuser interference (second-term of (1)). This means that the signal-tointerference-plus-noise-ratio translates to a SNR [5]. Note that $\mathbf{g}_{\ell}$ forms the $\ell$-th column of $\mathbf{G}=\mathbf{H}^{H}\left(\mathbf{H H}^{H}\right)^{-1}$, where $\mathbf{H}=\left[\mathbf{h}_{1}^{T}, \mathbf{h}_{2}^{T}, \ldots, \mathbf{h}_{L}^{T}\right]^{T}$ is the $L \times M$ matrix containing channels for all $L$ terminals. Recognizing that $\mathbf{H G}=\mathbf{H} \mathbf{H}^{H}\left(\mathbf{H H}^{H}\right)^{-1}=\mathbf{I}_{L}$, the ZF SNR at terminal $\ell$ is

$$
\mathrm{SNR}_{\ell}^{\mathrm{ZF}}=\frac{\rho_{\mathrm{t}} \beta_{\ell}}{\sigma^{2} \eta}=\frac{\rho_{\mathrm{t}} \beta_{\ell}}{\sigma^{2}\left\{\frac{1}{L}\left\{\operatorname{Tr}\left[\left(\mathbf{H H}^{H}\right)^{-1}\right]\right\}\right\}},
$$

since $\eta=\|\mathbf{G}\|_{F}^{2} / L=\operatorname{Tr}\left[\left(\mathbf{H H}^{H}\right)^{-1}\right] / L$. The ZF SNR in (2) can be used to estimate the ergodic sum spectral efficiency (in bits/sec/Hz) for all $L$ terminals. This is given by

$$
\mathrm{R}_{\mathrm{ZF}}=\mathbb{E}\left\{\sum_{\ell=1}^{L} \log _{2}\left(1+\mathrm{SNR}_{\ell}^{\mathrm{ZF}}\right)\right\},
$$

where the expectation is performed over small-scale fading. Below, closed-form approximations of (2) and (3) are derived.

\section{Analytical Results And Implications}

\section{A. Expected ZF SNR and Ergodic Sum Spectral Efficiency}

Remark 2. Finding exact moments of the ZF SNR in (2) is an extremely challenging task, since the matrix trace in its denominator is a random function of the inverse, of a complex non-standard semi-correlated central Wishart distribution formed by $\mathbf{H H}^{H}$. Moreover, $\mathbf{H}$ has a fully heterogeneous structure, since it contains $L$ different correlation patterns and link gains. Due to these reasons, we approximate the inverse in (2) with a finite order Neumann series expansion [15].

To do this, we separate $\mathbf{H H}^{H}$ into its expected diagonal components and correction terms. That is,

$$
\mathbf{H H}^{H}=M \mathbf{I}_{L}+\boldsymbol{\Delta},
$$


where $\boldsymbol{\Delta}=\mathbf{H H}^{H}-M \mathbf{I}_{L}$ and $\mathbb{E}\{\boldsymbol{\Delta}\}=\mathbf{0}$. Then, with an order $N$ Neumann series, one can approximate $\left(\mathbf{H H}^{H}\right)^{-1}$ as

$$
\left(\mathbf{H H}^{H}\right)^{-1} \approx \frac{1}{M} \sum_{n=0}^{N}(-1)^{n}\left(\frac{\boldsymbol{\Delta}}{M}\right)^{n} .
$$

Substituting the definition of $\boldsymbol{\Delta}$, and simplifying yields

$$
\begin{aligned}
\left(\mathbf{H H}^{H}\right)^{-1} & \approx \frac{1}{M} \sum_{n=0}^{N} \frac{(-1)^{n}}{(M)^{n}} \sum_{q=0}^{n}\left(\begin{array}{l}
n \\
q
\end{array}\right)\left(\mathbf{H H}^{H}\right)^{q}(-M)^{n-q} \\
& =\frac{1}{M} \sum_{n=0}^{N} \sum_{q=0}^{n}\left(\begin{array}{l}
n \\
q
\end{array}\right) \frac{(-1)^{q}}{(M)^{q}}\left(\mathbf{H} \mathbf{H}^{H}\right)^{q} .
\end{aligned}
$$

Substituting the above approximation into the denominator of (2) allows us to write the ZF SNR for terminal $\ell$ as

$$
\operatorname{SNR}_{\ell}^{\mathrm{ZF}} \approx \frac{\rho_{\mathrm{t}} \beta_{\ell}}{\sigma^{2}\left\{\frac{1}{L}\left\{\operatorname{Tr}\left[\frac{1}{M} \sum_{n=0}^{N} \sum_{q=0}^{n}\left(\begin{array}{l}
n \\
q
\end{array}\right) \frac{(-1)^{q}}{(M)^{q}}\left(\mathbf{H} \mathbf{H}^{H}\right)^{q}\right]\right\}\right\}} \text {. }
$$

Remark 3. In what follows, we evaluate the expected value of (7). Since this expectation needs to be performed over small-scale fading in $\mathbf{H H}^{H}$, which is in the denominator of (7), it is extremely cumbersome to evaluate. To overcome this difficulty, we employ the univariate special case of the commonly used first-order Laplace approximation [5, 16], allowing us to express (7) as (8) (shown on top of the following page for the reasons of space). The approximation in (8) is a first-order Laplace expansion and is of the form $\mathbb{E}\{\gamma / X\} \approx \gamma / \mathbb{E}\{X\}$, where $\gamma$ is a scalar value. As shown in $[5,16]$, the accuracy of such approximations relies on $X$ having a small variance relative to its mean. This can be seen by applying a multivariate Taylor series expansion to $\gamma / X$ around $\gamma / \mathbb{E}\{X\}$. The terms in (8) are well suited to this approximation, especially when $M$ and $L$ start to grow, since the implicit averaging in the denominator gives rise to the variance reduction required. In the following proposition, with a two-term Neumann series (i.e., $N=2$ ), a closed-form solution to (8) is presented for this heterogeneous system.

Proposition 1. When $\mathbf{h}_{\ell} \sim \mathcal{C N}\left(\mathbf{0}, \mathbf{R}_{\ell}\right)$, where $\mathbf{R}_{\ell}$ is the correlation matrix specific to terminal $\ell$, the expected $Z F S N R$ for the $\ell-$ th terminal can be approximated as

$$
\mathbb{E}\left\{\mathrm{SNR}_{\ell}^{\mathrm{ZF}}\right\} \approx \frac{\rho_{\mathrm{t}} \beta_{\ell} M^{3}}{\sigma^{2}\left\{L\left[M^{2}+L\left(\operatorname{Tr}\left[\overline{\mathbf{R}}^{2}\right]\right)\right]\right\}},
$$

where $\overline{\mathbf{R}}=\frac{\sum_{\ell=1}^{L} \mathbf{R}_{\ell}}{L}$, and is the average correlation matrix of all terminals in the system.

Proof: From (6), when $N=2$, one can write

$$
\left(\mathbf{H} \mathbf{H}^{H}\right)^{-1} \approx \frac{1}{M}\left[3 \mathbf{I}_{L}-\frac{3}{M} \mathbf{H H}^{H}+\frac{1}{M^{2}}\left(\mathbf{H} \mathbf{H}^{H}\right)^{2}\right] .
$$

Taking the matrix trace of (10) yields

$$
\begin{aligned}
& \operatorname{Tr}\left[\left(\mathbf{H H}^{H}\right)^{-1}\right] \approx \frac{1}{M}\left\{3 L-\frac{3}{M} \operatorname{Tr}\left[\mathbf{H} \mathbf{H}^{H}\right]\right. \\
&\left.+\frac{1}{M^{2}} \operatorname{Tr}\left[\left(\mathbf{H} \mathbf{H}^{H}\right)^{2}\right]\right\} .
\end{aligned}
$$

Performing the expectation of (11) results in taking the expec- tation of the individual terms on the right-hand side (RHS) of (11). As $\mathbb{E}\left\{\operatorname{Tr}\left[\mathbf{H H}^{H}\right]\right\}=M L$, the first two-terms on the RHS of (11) result in a cancellation, allowing us to focus on the expectation of the final term of (11). By definition, the final term on the RHS of (11) is given by

$$
\operatorname{Tr}\left[\left(\mathbf{H H}^{H}\right)^{2}\right]=\sum_{\ell=1}^{L} \sum_{j=1}^{L} \mathbf{h}_{\ell} \mathbf{h}_{j}^{H} \mathbf{h}_{j} \mathbf{h}_{\ell}^{H} .
$$

Taking the expectation of (12) over small-scale fading yields

$$
\begin{aligned}
& \mathbb{E}\left\{\operatorname{Tr}\left[\left(\mathbf{H H}^{H}\right)^{2}\right]\right\}=\mathbb{E}\left\{\sum_{\ell=1}^{L}\left(\mathbf{h}_{\ell} \mathbf{h}_{\ell}^{H}\right)^{2}+\sum_{\substack{\ell=1 \\
j=1 \\
j \neq \ell}}^{L} \sum_{\substack{j \\
\mathbf{h}_{\ell}}} \mathbf{h}_{j}^{H} \mathbf{h}_{j} \mathbf{h}_{\ell}^{H}\right\} \\
& =\mathbb{E}\left\{\sum_{\ell=1}^{L}\left\{M^{2}+\operatorname{Tr}\left[\mathbf{R}_{\ell}^{2}\right]\right\}+\sum_{\ell=1}^{L} \sum_{\substack{j=1 \\
j \neq \ell}}^{L} \operatorname{Tr}\left[\mathbf{R}_{\ell} \mathbf{R}_{j}\right]\right\} .
\end{aligned}
$$

Further simplifying the above expression allows us to state

$$
\begin{aligned}
\mathbb{E}\left\{\operatorname{Tr}\left[\left(\mathbf{H H}^{H}\right)^{2}\right]\right\} & =L M^{2}+\operatorname{Tr}\left[\sum_{\ell=1}^{L} \mathbf{R}_{\ell} \sum_{j=1}^{L} \mathbf{R}_{j}\right] \\
& =L\left\{M^{2}+L\left(\operatorname{Tr}\left[\overline{\mathbf{R}}^{2}\right]\right)\right\} .
\end{aligned}
$$

Inserting (14) into the mean of (11), and simplifying gives

$$
\mathbb{E}\left\{\operatorname{Tr}\left[\left(\mathbf{H H}^{H}\right)^{-1}\right]\right\} \approx \frac{L}{M^{3}}\left\{M^{2}+L\left(\operatorname{Tr}\left[\overline{\mathbf{R}}^{2}\right]\right)\right\} .
$$

The result in (15) can now be substituted into the denominator of (8) with some routine simplifications to obtain (9).

\section{B. Implications of Proposition 1}

To the best of the authors' knowledge, the result in (9) is the first, simple, closed-form approximation with ZF precoding and correlation diversity. The structure of (9) readily demonstrates the impact of correlation diversity via the term $\operatorname{Tr}\left[\overline{\mathbf{R}}^{2}\right]$, which influences the expected noise power of the desired terminal. Fixing all system parameters, one can observe that the expected ZF noise power is maximized when $\operatorname{Tr}\left[\overline{\mathbf{R}}^{2}\right]$ is maximized. By definition, $\operatorname{Tr}\left[\overline{\mathbf{R}}^{2}\right]=M+2 \sum_{i=1}^{M-1} \sum_{j=i+1}^{M}\left|\bar{r}_{i, j}\right|^{2}$, where $\bar{r}_{i, j}$ denotes the $(i, j)$-th element of $\mathbf{R}$, such that $\left|\bar{r}_{i, j}\right|^{2}=\left|\frac{1}{L} \sum_{\ell=1}^{L}\left(\mathbf{R}_{\ell}\right)_{i, j}\right|^{2}$. Maximizing $\left|\bar{r}_{i, j}\right|^{2}$ requires alignment of all the terms in the modulus. Specifically, the phases of all entries in $\mathbf{R}_{\ell}$ need to align in the $(i, j)$-th position, $\forall \ell=1,2, \ldots, L$. While such a scenario is generally unlikely to occur in practice, we identify one possible situation when this may take place: Precisely, when each terminal's correlation matrix is identical (the case for homogeneous channels), all phases will be aligned across all terminals in the $(i, j)$-th position. Though the above condition does not require the amplitudes of each terminal's correlation matrix to be equal, in the case of identical correlation matrices, the amplitudes will naturally also be equal across all terminals. Hence, identical correlation matrices result in the lowest ZF SNRs, serving as a useful lower bound for the performance of correlated multiuser channels. On the other hand, when each terminal experiences the same angular spread, yet a different mean 


$$
\mathbb{E}\left\{\mathrm{SNR}_{\ell}^{\mathrm{ZF}}\right\} \approx \frac{\rho_{\mathrm{t}} \beta_{\ell}}{\sigma^{2}\left\{\frac{1}{L}\left\{\frac{1}{M} \sum_{n=0}^{N} \sum_{q=0}^{n}\left(\begin{array}{l}
n \\
q
\end{array}\right) \frac{(-1)^{q}}{(M)^{q}} \mathbb{E}\left\{\operatorname{Tr}\left[\left(\mathbf{H H}^{H}\right)^{q}\right]\right\}\right\}\right.} .
$$

angle, $\operatorname{Tr}\left[\overline{\mathbf{R}}^{2}\right]$ yields a smaller value, since diversity is induced via the differences in the mean DOAs. On a similar note, with variations in both the angular spread and mean DOAs, maximum diversity kicks in, where $\operatorname{Tr}\left[\overline{\mathbf{R}}^{2}\right]$ tends to be even smaller, leading to a higher ZF SNR. In addition to the above, holding all other propagation and system parameters constant, increasing the number of BS antennas, $M$, causes a linear increase in the expected SNR (due to its numerator containing $M^{3}$ and the denominator containing a $M^{2}$ ). Meanwhile, increasing the number of user terminals, $L$, while keeping other parameters fixed leads to a quadratic increase in the expected noise power, degrading the ZF SNR. The above insights are difficult to obtain from more complex solutions derived in the literature (see e.g., $[8,12,13]$ ), which require a linked set of equations, even in the large system regime. In contrast, our analysis poses no such constraints and is general to the operational system dimension, and channel correlation structure. Note that (9) can be used to approximate the ergodic sum spectral efficiency by

$$
\mathbb{E}\left\{\mathrm{R}_{\mathrm{ZF}}\right\} \approx \sum_{\ell=1}^{L} \log _{2}\left(1+\mathbb{E}\left\{\mathrm{SNR}_{\ell}^{\mathrm{ZF}}\right\}\right) .
$$

The numerical accuracy of the derived results in (9) and (16) with their simulated counterparts is presented in Sec. V. In addition to the results presented in Sec. V, we note that (9) and (16) were explicitly tested with sample simulations based on scattering cluster circles in the propagation channel (one circle per-terminal) with varying center distances from each other. The one-ring correlation model described in Sec. V-A was used for the aforementioned evaluations, where agreement within $1 d B$ was found from the results derived in (9) and (16) relative to the simulated cases.

\section{Channel Measurements}

The propagation measurements were conducted in the oldtown city center in Cologne, Germany. The investigated area was mostly made up of buildings with similar heights and multiple floors (ranging between 4-8). The BS array (referred to as TX) was mounted on a rooftop of a $30 \mathrm{~m}$ building with the terminals (referred to as RX) placed on the rooftop of a car, at approximately $2.5 \mathrm{~m}$ above ground. The TX and RX placements in the environment are shown in Figs. 1 and 2. The measurements were conducted in 45 random terminal positions throughout the environment with a fixed TX array. To avoid interference at the desired frequencies, the German service provider, Deutsche Telekom, agreed to switch off their BSs operating at the same or adjacent frequency bands throughout the measurement duration. The measurements were performed with a wideband MIMO MEDAV RUSK channel sounder, operating at a center frequency of $2.53 \mathrm{GHz}$. The precise channel sounder settings are provided in [17]. The measurement setup utilizes cylindrical/circular array structures at both ends of the channel sounder. The array structures guarantee a truly three-dimensional (azimuth and zenith) channel measurement and ensures that MPCs from all directions in the urban environment could be easily captured. Naturally, both elevation and azimuth parameters need to be estimated to obtain accurate results, although in this paper only the azimuth parameters are used to populate the correlation models. The sounder is based on a combination of the switched array principle $[17,18]$ and virtual arrays. Only a single transmit and receive chain exist, where the transmit signal is connected via a fast electronic switch to elements of the TX array sequentially. The RX side operates in a similar manner to the TX, allowing for sequential measurement of the channel transfer function between all combinations of TX and RX antenna elements. The size of the TX array is increased by mechanical movement (rotation) of the physical elements. Overall, this process results in $60 \times 8 \times 2$ TX channels. This is equivalent to a 480 element cylindrical array operating with two polarization states. At the $\mathrm{RX}$, a stacked polarimetric uniform circular patch array with 2 (vertical) $\times 8$ (circumferal) $\times 2$ (polarization) antenna ports was employed. A detailed discussion on the above is provided in [17]. As long as the measurement sequence occurs within a timescale shorter than the channel coherence time, such a measurement is equivalent to a truly real-time measurement with parallel TX/RX chains for all antenna elements.

The RUSK sounder provides a 4-dimensional channel transfer function matrix, $\mathbf{H}(s, f, t, r)$, where $s$ denotes the measurement snapshot index, $f$ is the measured frequency index, while $t$ and $r$ denote TX and RX element indices, respectively. In total, $s=10$ snapshots were recorded for each measured transfer function to improve the measurement SNR when averaged. The impulse response of the propagation channel with a given $(t, r)$ pair was obtained via an inverse fast Fourier transform. To extract the spatial parameters of the propagation channel, a high resolution parameter estimation algorithm known as RIMAX was utilized [19]. RIMAX provides a complete double-directional description of the propagation channel [20], and extracts the spatial parameters to obtain an antenna independent characterization of the channel. This means that the spatial parameters of the channel remain independent to the type of the antenna array that is used to make the measurement. Due to space limitations, we omit presenting the procedure for identifying MPCs which belong to a particular cluster of scatterers. More details on this can be found in [17]. Leveraging this property, from the MPCs, we extract the root mean square azimuth angular spread of departure, as well as the mean DOA distributions across all 45 terminal positions. These form the basis for parameterizing the correlation structures considered in the paper (See Sec. V-A). Even though this is an approximation, such an approach is able to offer significantly greater insights than a purely numerical calculation of the correlation structure, as done routinely in the MU-MIMO literature. The extracted results are presented in Fig. 3 as cumulative distribution functions 


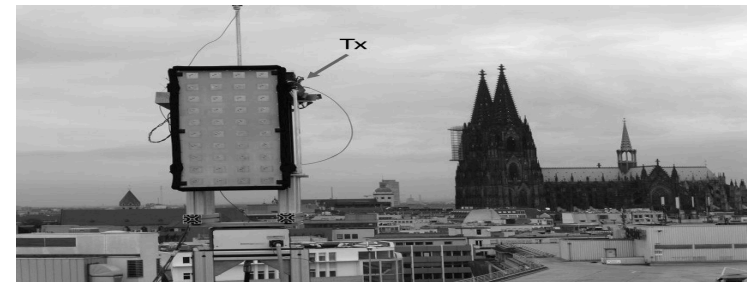

Fig. 1. BS array view of the UMa site in Cologne.

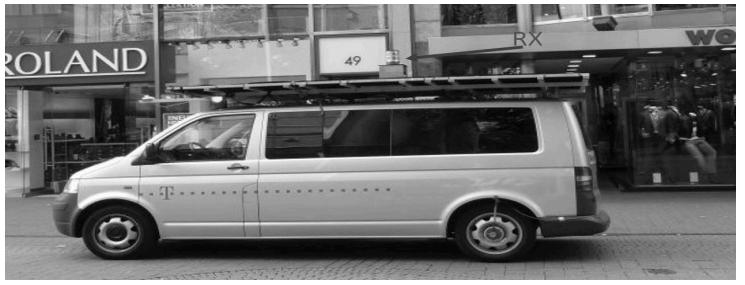

Fig. 2. Terminal placement in the coverage of the UMa site in Cologne.

(CDFs). It can be observed that the azimuth DOD spread has a degree of symmetry and spans over $40^{\circ}$. The variability in the angular spread is due to variability in the local scattering around the terminals, which can be modeled as $\mathcal{N}\left(14.02^{\circ},\left(6.45^{2}\right)^{\circ}\right)($ Gaussian fit on the fig.). In contrast to this, the mean DOA is $\mathcal{U}\left[-180^{\circ}, 180^{\circ}\right]$ (uniform fit on the fig.), primarily reflecting the distribution of the terminals in the measurement environment.

\section{NumERICAL RESUlTS}

Unless otherwise specified, the parameters described below are utilized for all numerical results, and are obtained from [21]. The cell radius, $R_{\mathrm{c}}=500 \mathrm{~m}$ was chosen with a reference distance $r_{0}=50 \mathrm{~m}$, such that terminals are randomly located outside $r_{0}$ and inside $R_{c}$, following $\mathcal{U}\left[-180^{\circ}, 180^{\circ}\right]$. Uniform power control is assumed so that the average transmit power is independent of distance. The UMa attenuation exponent of $\alpha=3.67$ was chosen. Furthermore, it is assumed that $\sigma^{2}=1$, and hence the average downlink SNR is equivalent to the average downlink transmit power, $\rho_{\mathrm{t}} / \sigma^{2}=\rho_{\mathrm{t}}$. The unitless constant for geometric attenuation, $A$, is chosen such that the fifth percentile of the instantaneous SNR with ZF precoding at terminal $\ell$ is $0 \mathrm{~dB}$, when $\rho_{\mathrm{t}}=0 \mathrm{~dB}$ with the baseline system parameters of $M=64$ and $L=6$. Note that the exponential correlation model (described later, with the correlation coefficient $\xi=0.9$ was used to obtain $A$. The shadowing standard deviation, $\sigma_{\mathrm{sh}}=6 \mathrm{~dB}$. For all numerical results, $10^{5}$ Monte-Carlo realizations were generated with an inter-element spacing, $d=0.5 \lambda$ at the BS, where applicable with $\lambda$ denoting the wavelength at the desired frequency.

\section{A. Channel Correlation Models}

As a baseline case, we assign a fixed correlation profile to each terminal with the widely used exponential model. Here, the $(i, j)$-th element of $\mathbf{R}_{\ell}$ is expressed as $\left[\mathbf{R}_{\ell}\right]_{i, j}=\xi^{|i-j|}$, for any $i, j$ in $1,2, \ldots, M$ with $0 \leq \xi \leq 1$ [5]. With correlation diversity, we employ two models, namely Cler$c k x$ [14], and one-ring [6,8,9] correlation. For the Clerckx correlation model, $\left[\mathbf{R}_{\ell}\right]_{i, j}=\xi_{\ell}^{|i-j|}$, where $\xi_{\ell}=|\xi| e^{j \Delta_{\ell}}$. Here, $|\xi|=\xi$, as in the exponential model, and is the same

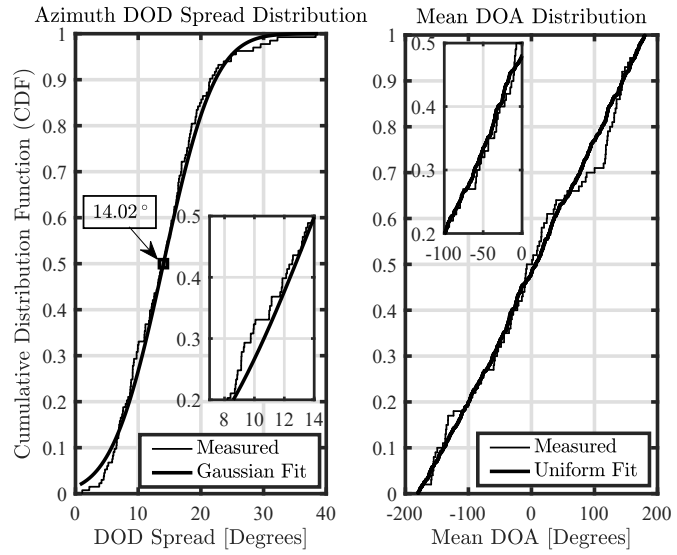

Fig. 3. Measured and fitted azimuth DOD spread and mean DOA CDFs at $2.53 \mathrm{GHz}$ in an UMa environment in Cologne.

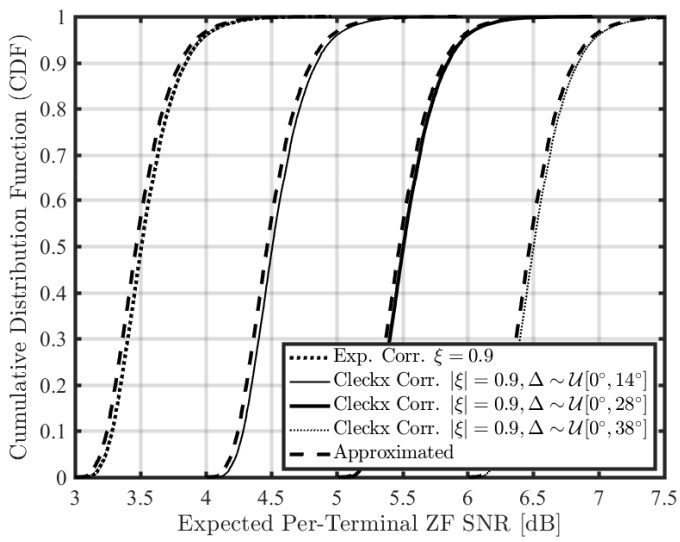

Fig. 4. CDFs of expected ZF SNR with $M=64, L=6$, and $\rho_{\mathrm{t}}=5 \mathrm{~dB}$.

for each terminal. However, a terminal specific phase, $\Delta_{\ell}$, is assumed to be $\mathcal{U}\left[-180^{\circ}, 180^{\circ}\right]$. This is used to differentiate the terminal locations. In each result, the range of $\Delta_{\ell}$ is specified. The one-ring model for terminal $\ell$ states $\left[\mathbf{R}_{\ell}\right]_{i, j}=\frac{1}{2 \Delta_{\ell}} \int_{-\Delta_{\ell}+\phi_{0}^{\ell}}^{\Delta_{\ell}+\phi^{\ell}} e^{-j 2 \pi d(i, j) \sin \left(\phi_{\ell}\right)} d \phi_{\ell}$, where $\Delta_{\ell}$ denotes the azimuth angular spread for terminal $\ell, \phi_{0}^{\ell}$ denotes the mean DOA, and $\phi_{\ell}$ is the actual DOA, uniformly distributed within the angular spread around the mean DOA. Furthermore, $d(i, j)$ captures the normalized antenna spacing between the $i$-th and $j$-th elements. The precise values of $\Delta_{\ell}$ for the onering model are specified in each subsequent result.

\section{B. Impact of Correlation Diversity}

Figure 4 depicts the CDFs of the expected ZF SNR with $M=64, L=6$, and $\rho_{\mathrm{t}}=5 \mathrm{~dB}$. Each density is obtained by averaging over the small-scale fading, with the CDFs representing the variations from the link gains. Two trends can be observed: Firstly, with diverse correlation matrices from the Clerckx model, the larger the spread of the random phases in $\Delta$ 's, the higher the expected ZF SNR. Despite the correlation magnitude being as high as $\xi=0.9$, increasing the spread of $\Delta$ to $\mathcal{U}\left[0,14^{\circ}\right], \mathcal{U}\left[0,28^{\circ}\right]$, and $\mathcal{U}\left[0,38^{\circ}\right]$ yields a $0.93,2$, and $3 \mathrm{~dB}$ gain in the expected ZF SNR, relative to the exponential model case. This performance difference is owed to the fact that increasing the spread of $\Delta$ increases the amount of spatial selectivity induced across multiple 


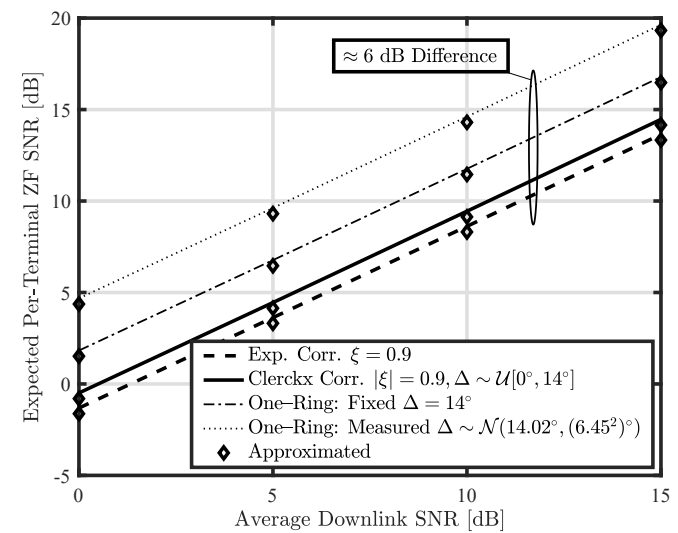

Fig. 5. Expected ZF SNR vs. average SNR with $M=64$ and $L=6$.

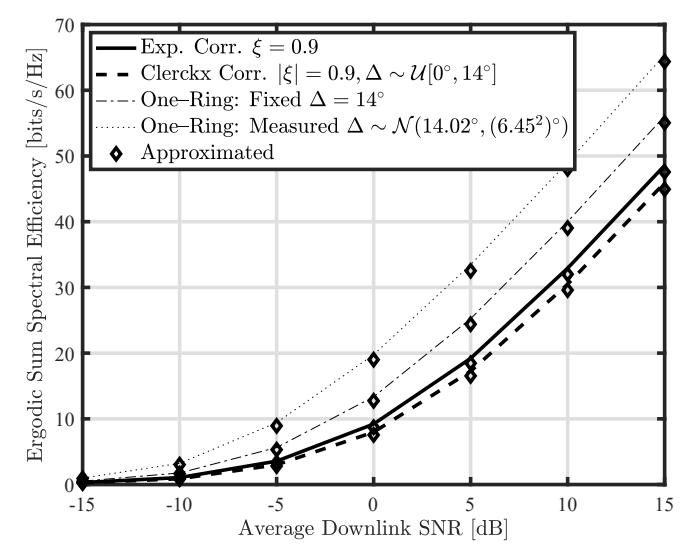

Fig. 6. ZF Ergodic sum spectral efficiency vs. average SNR with $M=128$ and $L=10$.

channels, allowing the composite channel rank to increase. The result demonstrates the sensitivity of multiuser channels to changes in the phase of the correlation matrices. Secondly, our proposed approximations are tight in comparison to the simulated cases for all $\Delta$ values. Figure 5 demonstrates the expected ZF SNR as a function of the average operating SNRs. Here, in addition to the cases for exponential correlation and Clerckx correlation, performance with the one-ring model is also evaluated. It can be observed that even with a fixed angular spread, the one-ring model predicts higher expected SNRs in comparison to the Clerckx model. This is due to the fact that both the magnitude and phase of the correlation matrices are variable across each terminal in the case of the one-ring model. Moreover, when evaluating the expected SNRs with the measured angular spread values, a further $3 \mathrm{~dB}$ increase in the ZF SNR is seen across all SNRs. This is mainly due to the increased diversity brought by the variable angular spreads (Gaussian random variables). The proposed approximations are seen to remain tight across all the considered models, and SNR values. Keeping all other parameters constant, Fig. 6 depicts the ZF ergodic sum spectral efficiency as a function of the operating SNR with $M=128$ and $L=10$. While similar trends to Fig. 5 can be observed, it can be seen that the remarkably simple approximations remain tight across a wider range of system dimensions and operational SNRs.

\section{CONCLUSIONS}

The paper presents closed-form approximations to the ZF expected SNR and ergodic sum spectral efficiency of a MU-
MIMO system. With unequally correlated Rayleigh fading, our analysis is robust to various physical and non-physical channel correlation models, as well as average downlink SNR. More physically motivated models, such as one-ring correlation, consider unequal magnitudes and phases in the correlation matrices for each terminal, and tend to estimate higher MU-MIMO performance. Data from the recent 2.53 $\mathrm{GHz}$ UMa propagation measurements was extracted to accurately parameterize correlation models in order to characterize their impact on MU-MIMO performance. Such an evaluation emphasizes the fact that the performance of a MU-MIMO system is ultimately governed by the correlation model, and its parameters in use.

\section{REFERENCES}

[1] X. Gao, et al., "Massive MIMO performance evaluation based on measured propagation data," IEEE Trans. Wireless Commun., vol. 14, no. 7, pp. 3899-3911, Jul. 2015.

[2] S. L. H. Nguyen, et al., "On the mutual orthogonality of millimeter-wave massive MIMO channels," in Proc. IEEE VTC-Spring, May 2015.

[3] X. Gao, et al., "Massive MIMO channels - measurements and models," in Proc. IEEE ASILOMAR, Dec. 2013, pp. 280-284.

[4] H. Falconet, et al., "Asymptotic analysis of downlink MISO systems over Rician fading channels," in Proc. IEEE ICASSP, May 2016, pp. 39263930.

[5] H. Tataria, et al., "On the general analysis of coordinated regularized zero-forcing precoding: An application to two-tier small-cell networks," IEEE Trans. Commun., vol. 65, no. 7, pp. 3133-3150, Jul. 2017.

[6] Z. Jiang, et al., "Achievable rates of FDD massive MIMO systems with spatial channel correlation," IEEE Trans. Wireless Commun., vol. 14, no. 5, pp. 2868-2882, May 2015.

[7] J. Nam, et al., "On the role of transmit spatial correlation diversity in multiuser MIMO systems," IEEE Trans. Inf. Theory, vol. 63, no. 1, pp. 336-354, Jan. 2017.

[8] A. Adhikary, et al., "Joint spatial division and multiplexing for mm-Wave channels," IEEE J. Sel. Areas Commun., vol. 32, no. 6, pp. 1239-1255, Jun. 2014.

[9] E. Bjornsön, et al., "Pilot contamination is not a fundamental asymptotic limitation in massive MIMO," in Proc. IEEE ICC, May 2017.

[10] J. Poutanen, et al., "Multi-link MIMO channel modeling using geometrybased approach," IEEE Trans. Antennas and Propag., vol. 60, no. 2, pp. 587-596, Feb. 2012.

[11] J. Iscar, et al., "Optimal angular spread of the multipath clusters in mmWave systems under pilot contamination," in Proc. IEEE VTC-Fall, Sep. 2017.

[12] J. Hoydis, et al., "Massive MIMO in the UL/DL of cellular networks: How many antennas do we need?,' IEEE J. Sel. Areas Commun., vol. 31, no. 2, pp. 160-171, Feb. 2013.

[13] S. Wagner, et. al., "Large system analysis of linear precoding in correlated MISO broadcast channels under limited feedback," IEEE Trans. Inf. Theory, vol. 58, no. 7, pp. 4509-4537, Jul. 2012.

[14] B. Clerckx, et al., "Correlated fading in broadcast MIMO channels: Curse or blessing?," in Proc. IEEE GLOBECOM, Nov. 2008.

[15] D. Zhu, et al., "On the matrix inversion approximation based on Neumann series in massive MIMO systems," in Proc. IEEE ICC, Jun. 2015.

[16] Q. Zhang, et al., "Power scaling of uplink massive MIMO systems with arbitrary-rank channel means," IEEE J. Sel. Topics Signal Process., vol. 8, no. 5, pp. 966-981, Oct. 2014.

[17] S. Sangodoyin, et al., "Cluster-based analysis of 3D MIMO channel measurement in an urban environment," in Proc. IEEE MILCOM, Oct. 2015, pp. 744-749.

[18] R. S. Thomä, et al., "Identification of time-variant directional mobile radio channels," IEEE Trans. Instrum. Meas., vol. 49, no. 2, pp. 357-364, Apr. 2000.

[19] A. Richter, "Estimation of radio channel parameters: Models and algorithms," Ph.D. dissertation, Technische Universität Ilmenau, Germany, 2005, Available online: www.db-thueringen.de.

[20] M. Steinbauer, et al., "The double-directional radio channel," IEEE Antennas and Propag. Mag., vol. 43, no. 4, pp. 51-63, Aug. 2001.

[21] 3GPP TR 36.873 v.12.2.0, Study on 3D channel models for LTE, 3GPP, Jun. 2015. 